\title{
Moving Horizon Estimation for Tire-Road Friction During Braking
}

\author{
Dan Sui and Tor A. Johansen
}

\begin{abstract}
Estimation of tire-road friction forces has an important role in anti-lock brake systems (ABS), as well as for vehicle stability control systems, and road condition monitoring systems. We investigate the use of a moving horizon observer for estimation of multiple friction model parameters as well as the longitudinal wheel slip state under typical ABS braking scenarios, using wheel speed measurement and information on the brake torque. It is well known that data may not be persistently exicting for every period of time in such scenarios, expecially when estimating several friction model parameters simultaneously. The algorithm therefore has regularization mechanisms to ensure graceful degradation of the state and parameter estimation performance in cases when data are not persistently exciting. Simulations with a quarter car dynamic model and the four-parameter longitudinal Magic-formula friction model illustrates the performance of the algorithm.
\end{abstract}

\section{INTRODUCTION}

An anti-lock brake system (ABS) controls the slip of each wheel of a vehicle to prevent it from locking such that high longitudinal and lateral friction is achieved and steerability is maintained during hard braking. ABS are characterized by robust adaptive behavior with respect to highly uncertain tyre characteristics and fast changing road surface properties and they have been commercially available in cars for 30 years ([1], [2]).

Since the vehicle forces transferred from the tires to the road determine the vehicle motion, accurate information about road surface properties (dry, wet, snow, ice, etc.) has a significant importance in ABS and other automotive active safety systems. However, such forces and road surface properties are usually difficult to measure, since sensors are too complex and expensive for use in production cars. Therefore, it is necessary to estimate them from the computed or measurable signals such as angular wheel speed and the torque acting on the wheel's axis. In order to take advantage of a friction model to estimate the longitudinal wheel slips and speed for use in a wheel slip control system, one should also estimate one or more parameters of the friction model [3], [4].

Previous work has considered the use of nonlinear Lyapunov-based observers to estimate multiple parameters of the LuGre friction model [4], [5], [6], [7], the use of Kalmanfiltering to estimate the maximum friction coefficient of the Magic formula and similar friction models [3], [8], [9], [10]. In this paper, a novel approach based on moving horizon

This work was supported by the Research Council of Norway under the Strategic University Program on Computational Methods in Nonlinear Motion Control.

D. Sui and T. A. Johansen are with Department of Engineering Cybernetics, Norwegian University of Science and Technology, Trondheim, Norway. tor.arne. johansenditk. ntnu. no estimation (MHE) is proposed to be used to estimate multiple parameters of the Magic formula longitudinal friction model [11]. MHE is a direct approach to the deterministic discretetime nonlinear state and parameter estimation problem. In its simplest form, it views the problem as one of inverting a sequence of nonlinear algebraic equations defined from the state update and measurement equations, and some moving time horizon [12]. The novel contribution of the present work is to provide and study an MHE method based on [13] which contains modifications of the approach [14] that aims give graceful degradation of performance also when the condition of uniform observability is violated due to temporary lack of peristently exciting input data. Given the data such as measured signals of angular speeds, and torque acting on wheel axis, the proposed MHE algorithm can provide useful estimation of road surface properties and wheel slip even in cases when the data has not much excitation. The algorithm uses a dynamic model augmented with artificial states for the unknown parameters to be estimated, and includes the use of regularizing a priori estimates in the moving horizon cost function, and the use of thresholded singular value decomposition to avoid ill-conditioned or ill-posed inversion of the associated nonlinear algebraic equations that define the moving horizon state estimate [13]. A standard variant of MHE was proposed for estimation of friction force and vehicle speed using the LuGre model in [15]. The present paper considers a dynamic quarter car model, the magic formula model, and utilizes an MHE algorithm with regularization in order to effectively deal with situations without persistence of excitation.

The following notation and nomenclature is used. For a vector $x \in \mathbb{R}^{n}$, let $\|x\|=\sqrt{x^{T} x}$ denote the Euclidean norm. Recall that the induced matrix norm $\|M\|$ equals the largest singular value of the matrix $M$. For two vectors $x \in \mathbb{R}^{n}$ and $y \in \mathbb{R}^{m}$, let $\operatorname{col}(x, y)$ denote the column vector in $\mathbb{R}^{n+m}$ where $x$ and $y$ are stacked into a single column. The Moore-Penrose pseudo-inverse ([16]) of a matrix $M$ is denoted $M^{+}$and for a matrix $M$ of full rank it is given by $M^{+}=\left(M^{T} M\right)^{-1} M^{T}$. The composition of two functions $f$ and $g$ is written $f \circ g(x)=$ $f(g(x))$. Finally, a function $\varphi: \mathbb{R}^{+} \rightarrow \mathbb{R}$ is called a $K$-function if $\varphi(0)=0$

\section{MOdel Description}

In this section, we review a mathematical model of the wheel slip dynamics, see also [1], [3]. The problem of wheel slip control is best explained by looking at a quarter car model as shown in Figure 1. The model consists of a single wheel attached to a mass $m$. As the wheel rotates, driven by the inertia of the mass $m$ in the direction of the velocity $v$, a 


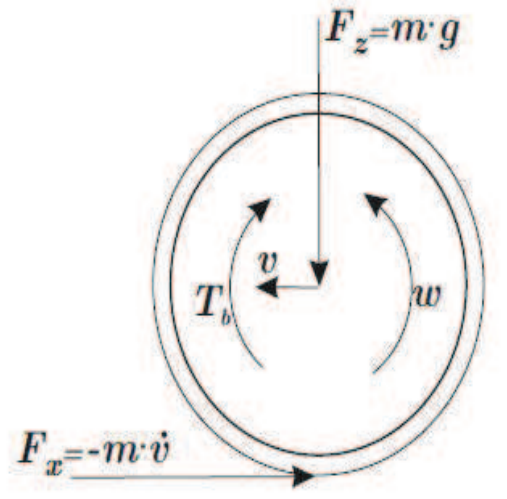

Fig. 1. Quarter car model forces and torques, [3].

\begin{tabular}{llll}
\hline Para. & Description & Value & Unit \\
\hline$m$ & Quarter vehicle mass. & 325 & $\mathrm{~kg}$ \\
$J$ & Moment of inertia of the wheel. & 1 & $\mathrm{kgm}$ \\
$r$ & Wheel radius. & 0.345 & $\mathrm{~m}$ \\
$g$ & Acceleration of gravity. & 9.81 & $\mathrm{~m} / \mathrm{s}^{2}$ \\
$F_{z}$ & Vertical force. & 3188 & $\mathrm{kgm} m^{2} / \mathrm{s}^{2}$ \\
$v$ & Longitudinal speed. & & $\mathrm{m} / \mathrm{s}$ \\
$\omega$ & Angular speed. & $\mathrm{rad} / \mathrm{s}$ \\
$\lambda$ & Longitudinal tyre slip. & $\mathrm{m} / \mathrm{s}$ \\
$T_{b}$ & Torque acting on wheel axis. & $\mathrm{Nm}$ \\
$F_{X}$ & Friction force between wheel and road. & $\mathrm{N}$ \\
$\theta$ & Road condition coefficient. & - \\
\hline
\end{tabular}

TABLE I

Model VAIABLES. THE NUMERIC VALUES ARE NOMINAL VALUES USED IN THE SIMULATION CASE STUDY.

tyre reaction force $F_{x}$ is generated by the friction between the tyre surface and the road surface. The tyre reaction force will generate a torque that results in a rolling motion of the wheel causing an angular velocity $\omega$. A brake torque applied to the wheel will act against the spinning of the wheel causing a negative angular acceleration. The equations of motion of the quarter car are [1], [3]

$$
\begin{aligned}
& m \dot{v}=-F_{x} \\
& J \dot{\omega}=r F_{x}-T_{b} \operatorname{sign}(\omega) .
\end{aligned}
$$

The parameters used in the paper are given in Table 1. The longitudinal tire friction force $F_{x}$ is given by the so-called magic formula [11]

$$
\begin{aligned}
F_{x}: & =F_{z} \mu(\lambda)=m g \mu(\lambda) \\
& =F_{z} \theta \sin (C(\arctan B \lambda-E(B \lambda-\arctan (B \lambda)))),
\end{aligned}
$$

where the friction coefficient $\mu$ is a nonlinear function of the longitudinal slip

$$
\lambda=\frac{v-\omega r}{v} .
$$

The parameters $B, C, E$ and $\theta$ characterize the tire and the road surface. Typical values of parameters $B, C, E$ and $\theta$ are given by [10] with

$$
B=10.38, C=1.65, E=0.65663, \theta=1
$$

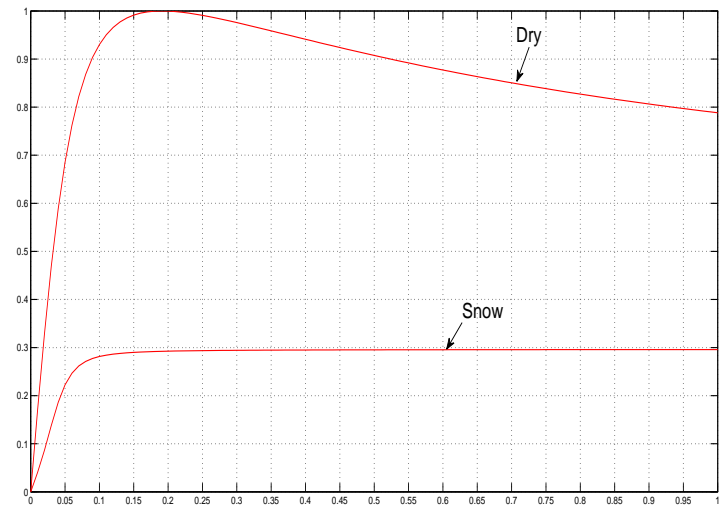

Fig. 2. Typical friction curves $\mu(\lambda)$.

for dry asphalt and

$$
B=14.395, C=0.9, E=-6.439, \theta=0.3
$$

for snow. The longitudinal slip $\lambda$ describes the normalized difference between the vehicle's longitudinal velocity $v$ and the speed of the wheel perimeter $\omega r$. The slip value of $\lambda=0$ characterizes the free motion of the wheel where no friction force $F_{x}$ is exerted $(v=\omega r)$. If the slip attains the value $\lambda=1$ then the wheel is locked $(\omega=0)$. The typical friction curves $\mu(\lambda)$ are shown in Figure 2. The friction coefficient $\mu$ is generally a differentiable function with the property $\mu(0)=0$ and $\mu(\lambda)>0$ for $\lambda>0$. Figure 2 shows how $\mu$ increases with slip $\lambda$ up to some value, where it attains its maximum value. For higher slip values, the friction coefficient will decrease to a minimum value where the wheel is locked and only the sliding friction will act on the wheel. The dependence of friction on the road condition is also exemplified in Figure 2. For wet or icy roads, the maximum friction is small and the right part of the curve is typically flatter.

Using (1)-(3), for $v>0$ and $\omega \geq 0$ we get the wheel slip dynamics

$$
\begin{aligned}
& \dot{v}=-\frac{1}{m} F_{z} \mu(\lambda), \\
& \dot{\lambda}=-\frac{1}{v}\left(\frac{1}{m}(1-\lambda)+\frac{r^{2}}{J}\right) F_{z} \mu(\lambda)+\frac{1}{v} \frac{r}{J} T_{b} .
\end{aligned}
$$

It is assumed that only wheel angular velocity $\omega$ is measurable while vehicle speed $v$ and the friction parameters need to be estimated. Thus the measurement equation is:

$$
y=\omega=\frac{v(1-\lambda)}{r} .
$$

We assume $T_{b}$ and $F_{z}$ are known, i.e. can be computed from information in the engine and ABS systems.

\section{Nonlinear Moving Horizon Estimator}

In this section we describe the MHE [13]. It is assumed that in the dynamic state space model, the state $x$ is augmented by constant parameters as agumented states, like 
$\dot{\theta}=0, \dot{\beta}=0, \ldots$ By discretization, it is assumed that the nonlinear system can be descried by the dynamics

$$
\begin{aligned}
x(t+1) & =f(x(t), u(t)) \\
y(t) & =h(x(t)),
\end{aligned}
$$

where $x(t) \in \mathbb{X} \subseteq R^{n_{x}}, u(t) \in \mathbb{U} \subseteq R^{n_{u}}$ and $y(t) \in R^{n_{y}}$ are respectively the augmented state vector, input and measurement vectors, and $t$ is the discrete time index. It is assumed that $\mathbb{X}$ and $\mathbb{U}$ are compact sets.

The $N+1$ consecutive measurements of outputs and inputs until time $t$ are denoted as $Y(t)=\operatorname{col}(y(t-N), y(t-N+$ $1), \cdots, y(t))$ and $U(t)=\operatorname{col}(u(t-N), u(t-N+1), \cdots, u(t-$ $1))$. To express $Y(t)$ as a function of $x(t-N)$ and $U(t)$, denote $f^{u(t)}(x(t))=f(x(t), u(t))$, and note from (8b) that the following algebraic map can be formulated, see [12]:

$$
\begin{aligned}
& Y(t)=H(x(t-N), U(t))=H_{t}(x(t-N)) \\
& =\left[\begin{array}{r}
h(x(t-N)) \\
h \circ f^{u(t-N)}(x(t-N)) \\
\vdots \\
h \circ f^{u(t-1)} \circ \cdots \circ f^{u(t-N)}(x(t-N))
\end{array}\right],
\end{aligned}
$$

where $\circ$ denotes composition of functions.

From [12], the system (8) is $N$-observable if there exists a $K$-function $\varphi$ such that for all $x_{1}, x_{2} \in \mathbb{X}$ there exists a feasible $U(t) \in \mathbb{U}^{N+1}$ such that

$$
\varphi\left(\left\|x_{1}-x_{2}\right\|^{2}\right) \leq\left\|H\left(x_{1}, U(t)\right)-H\left(x_{2}, U(t)\right)\right\|^{2} .
$$

From [13], the input $U(t) \in \mathbb{U}^{N+1}$ is said to be $N$-exciting for the $N$-observable system (8) at time $t$ if there exists a $K$-function $\varphi_{t}$ such that for all $x_{1}, x_{2} \in \mathbb{X}$

$$
\varphi_{t}\left(\left\|x_{1}-x_{2}\right\|^{2}\right) \leq\left\|H\left(x_{1}, U(t)\right)-H\left(x_{2}, U(t)\right)\right\|^{2} .
$$

Next, define the $N$-information vector at time $t$ as

$$
I(t)=\operatorname{col}(y(t-N), \ldots, y(t), u(t-N), \ldots, u(t-1))(10)
$$

The problem consists in estimating, at any time $t=N, N+$ $1, \ldots$, the state vectors $x(t-N), \ldots, x(t)$, on the basis of a priori estimates $\bar{x}(t-N, t), \ldots, \bar{x}(t, t)$ and the information vector $I(t)$. It is assumed that an a priori estimator is determined from the last estimate $\hat{x}^{o}(t-N-1, t-1)$ via the application of the function $f$, that is,

$$
\bar{x}(i+1, t)=f(\bar{x}(i, t), u(i)), \quad i=t-N, \ldots, t-1,
$$

where $\bar{x}(t-N, t)=f\left(\hat{x}^{o}(t-N-1, t-1), u(t-N-1)\right)$. A convergent estimate is computed by minimizing the following weighted regularized least-squares criterion with respect for $\hat{x}(t-k, t), k=0, \ldots, N$ :

$$
\begin{gathered}
J(\hat{x}(t-N, t), \bar{x}(t-N, t), I(t))=\left\|W_{t}\left(Y(t)-H_{t}(\hat{x}(t-N, t))\right)\right\|^{2} \\
+\sum_{i=t-N}^{i=t} \beta_{i-t+N}\|\hat{x}(i, t)-\bar{x}(i, t)\|^{2}
\end{gathered}
$$

subject to

$$
\begin{aligned}
\hat{x}(i+1, t) & =f(\hat{x}(i, t), u(i)), \quad i=t-N, \ldots, t-1, \\
\hat{x}(i, t) & \in \mathbb{X}, \quad i=t-N, \ldots, t,
\end{aligned}
$$

with $\beta_{i-t+N} \geq 0$, and $W_{t}$ being a time-varying weight matrix. As discussed in [13], tuning of $W_{t}$ and $\beta_{i} \geq 0$ should consider the tradeoff between minimizing the effects of measurement noise, model errors, and possibly other uncertainty. In addition, it might be beneficial to consider the information contents in the data at any time $t$, since an acceptable tuning may have to change with time to adaptively account for lack of persistently exciting data.

For $N$-observable systems, it is shown in [13] that the convergence condition will depend on the existence of a (not too small) $\varepsilon>0$ such that

$$
\Phi_{t}^{T}\left(x(t-N), \hat{x}^{o}(t-N, t)\right) \Phi_{t}\left(x(t-N), \hat{x}^{o}(t-N, t)\right) \geq \varepsilon I>0
$$

for all $t \geq 0$, where

$$
\begin{aligned}
& \Phi_{t}\left(x(t-N), \hat{x}^{o}(t-N, t)\right) \\
& =\int_{0}^{1} \frac{\partial}{\partial x} H\left((1-s) x(t-N)+s \hat{x}^{o}(t-N, t), U(t)\right) d s .
\end{aligned}
$$

This condition comes from the requirement of the input data being $N$-exicting at all $t$ and is similar to a persistence of excitation condition commonly seen in adaptive observers and estimators. Unfortunately, since $\Phi_{t}\left(x(t-N), \hat{x}^{o}(t-N, t)\right)$ depends on the unknown $x(t-N)$ we cannot monitor directly if $U(t)$ is sufficiently rich. Instead, we have to rely on some approximation or estimate of $\Phi_{t}(\cdot)$. If it is assumed that the initial estimation error is small, then

$$
\begin{aligned}
& \Phi_{t}\left(x(t-N), \hat{x}^{o}(t-N, t)\right) \approx \Phi_{t}\left(\hat{x}^{o}(t-N, t), \hat{x}^{o}(t-N, t)\right) \\
& =\frac{\partial H}{\partial x}\left(\hat{x}^{o}(t-N, t), U(t)\right):=\hat{\Phi}_{t} .
\end{aligned}
$$

Consider a singular value decomposition (SVD)([16])

$$
\hat{\Phi}_{t}=U_{t} S_{t} V_{t}^{T} .
$$

Any singular value (diagonal element of the matrix $S_{t}$ ) that is zero or close to zero indicates that a component is unobservable or the input is not $N$-exciting. Moreover, the corresponding row of the $V_{t}$ matrix will indicate which components are not $\mathrm{N}$-observable or $\mathrm{N}$-exciting. The $\mathrm{N}$ excitation of data may therefore be monitored through the robust computation of the rank of the Jacobian matrix using the SVD ([16]).

In [13] it is proposed to choose $W_{t}$ such that

$$
\left\|\left(W_{t} U_{t} S_{t} V_{t}^{T}\right)^{+}\right\|= \begin{cases}\alpha, & \text { if }\left\|S_{t}\right\| \geq \delta \\ 0, & \text { otherwise }\end{cases}
$$

where $\alpha>0$ is a scalar tuning parameter, and $\delta \geq 0$ is a regularization parameter. This leads to

$$
W_{t}=(1 / \alpha) V_{t} S_{\delta, t}^{+} U_{t}^{T}
$$

satisfying the following sufficient condition for local exponential convergence of the estimation error [13]:

$$
\left\|\left(W_{t} \hat{\Phi}_{t}\right)^{+}\right\| \leq \alpha .
$$

provided that $\alpha>0$ is sufficiently small, and the thresholded pseudo-inverse $S_{\delta, t}^{+}=\operatorname{diag}\left(1 / \sigma_{1, t}, \ldots, 1 / \sigma_{\ell, t}, 0, \ldots, 0\right)$ where $\sigma_{1}, \ldots, \sigma_{\ell}$ are the singular values larger than some $\delta>0$ and 
the zeros correspond to small singular values whose inverse is set to zero ([16]). The tuning parameters with this adaptive choice of $W_{t}$ are the scalars $\alpha, \delta$ and $\beta_{i}$. It is worthwhile to notice that since they are scalars, a successful tuning of the observer depends in general on appropriate scaling of the data, states and model equations although in this case study no particular scaling was found necessary to acceptable performance.

\section{Simulation RESUlts}

In this section, the regularized MHE algorithm is applied to the combined state and parameter estimation problem of estimating $v, \lambda$ and a number of friction model parameters. The simulation were performed using the model (6). Suppose $v(0)=20, \lambda(0)=0.01$, and $\theta, B, C$ and $E$ are given in (4) and (5) according to the different scenarios. In the simulation, choose the initial a priori estimate $\bar{v}(0)=19, \bar{\lambda}(0)=0$ and choose a set of nominal values of $\bar{\theta}, \bar{B}, \bar{C}$ and $\bar{E}$ as

$$
\bar{\theta}=0.6, \bar{B}=12, \bar{C}=1.3, \bar{E}=0
$$

which are used in all scenarios, either as the initial a priori estimate if the corresponding parameter needs to be estimated or as the fixed value if the corresponding parameter is not estimated. The horizon is chosen as $N=10$. The sampling interval $t_{f}=0.01 \mathrm{~s}$, and Gaussian white noise with variance $0.2 \mathrm{rad} / \mathrm{s}$ is added to the wheel speed measurements. In the figures, true states are shown in solid lines and estimated states are shown in dash lines.

\section{A. Tyre/road condition coefficient $\theta$ is unknown}

We consider the road condition coefficient $\theta$ as an augmented state, and get the augmented wheel slip dynamics

$$
\begin{aligned}
\dot{v} & =-\frac{1}{m} F_{z} \mu(\lambda, \theta), \\
\dot{\lambda} & =-\frac{1}{v}\left(\frac{1}{m}(1-\lambda)+\frac{r^{2}}{J}\right) F_{z} \mu(\lambda, \theta)+\frac{1}{v} \frac{r}{J} T_{b}, \\
\dot{\theta} & =0 .
\end{aligned}
$$

The following constraints are imposed:

$$
v(t) \geq 0,0 \leq \lambda(t) \leq 1,0 \leq \theta(t) \leq 1 .
$$

Choose $\alpha=0.01$ and $\beta_{i}=1$, and $W_{t}$ is chosen according to (16) with $\delta=0.1$.

- Case 1 , for dry asphalt, the simulation result is shown in Figure 3.

- Case 2, for snow, the simulation result is shown in Figure 4.

We observe that although the estimate of the single parameter $\theta$ converges to an accurate estimate, the observer still fails in estimating the velocity and wheel slip in Case 2 due to the inaccurate fixed values of $\bar{B}, \bar{C}$ and $\bar{E}$ used in the model. Hence, there is a potential benefit of estimating also $B, C$ and E.
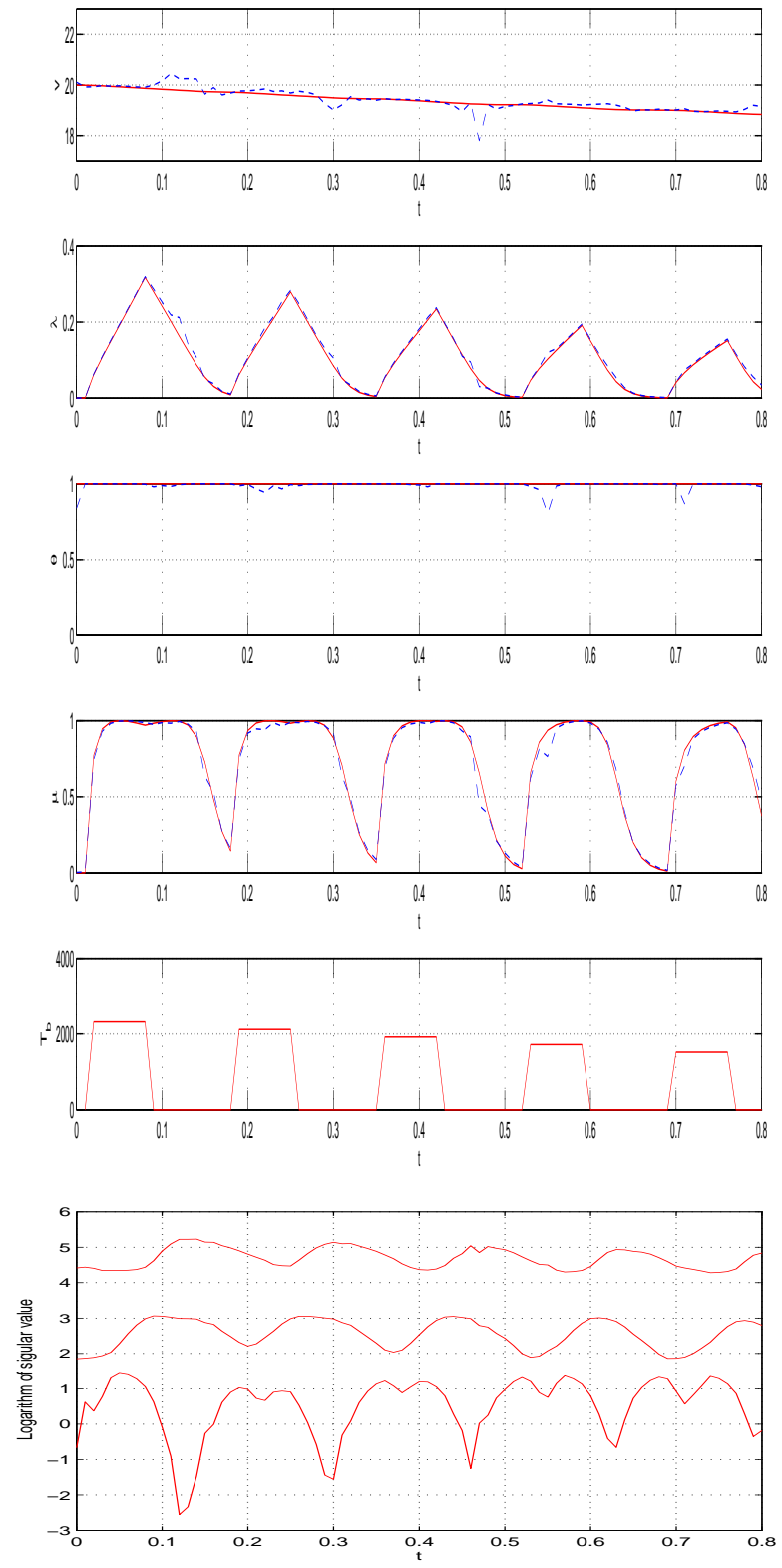

Fig. 3. Simulation results of case 1 .

\section{B. $\theta, E, C, B$ are unknown}

Considering the parameters $\theta, E, C, B$ as augmented states

$$
\dot{B}=0, \dot{C}=0, \dot{E}=0 .
$$

The constraints of $B, C$ and $E$ are given as

$$
\begin{aligned}
9 & \leq B(t) \leq 15.5 \\
0 & \leq C(t) \leq 3 \\
-7.5 & \leq E(t) \leq 2
\end{aligned}
$$

Choose $\alpha=0.01$ and $\beta_{i}=1 . W_{t}$ is chosen according to (16) with $\delta=0.8$. 

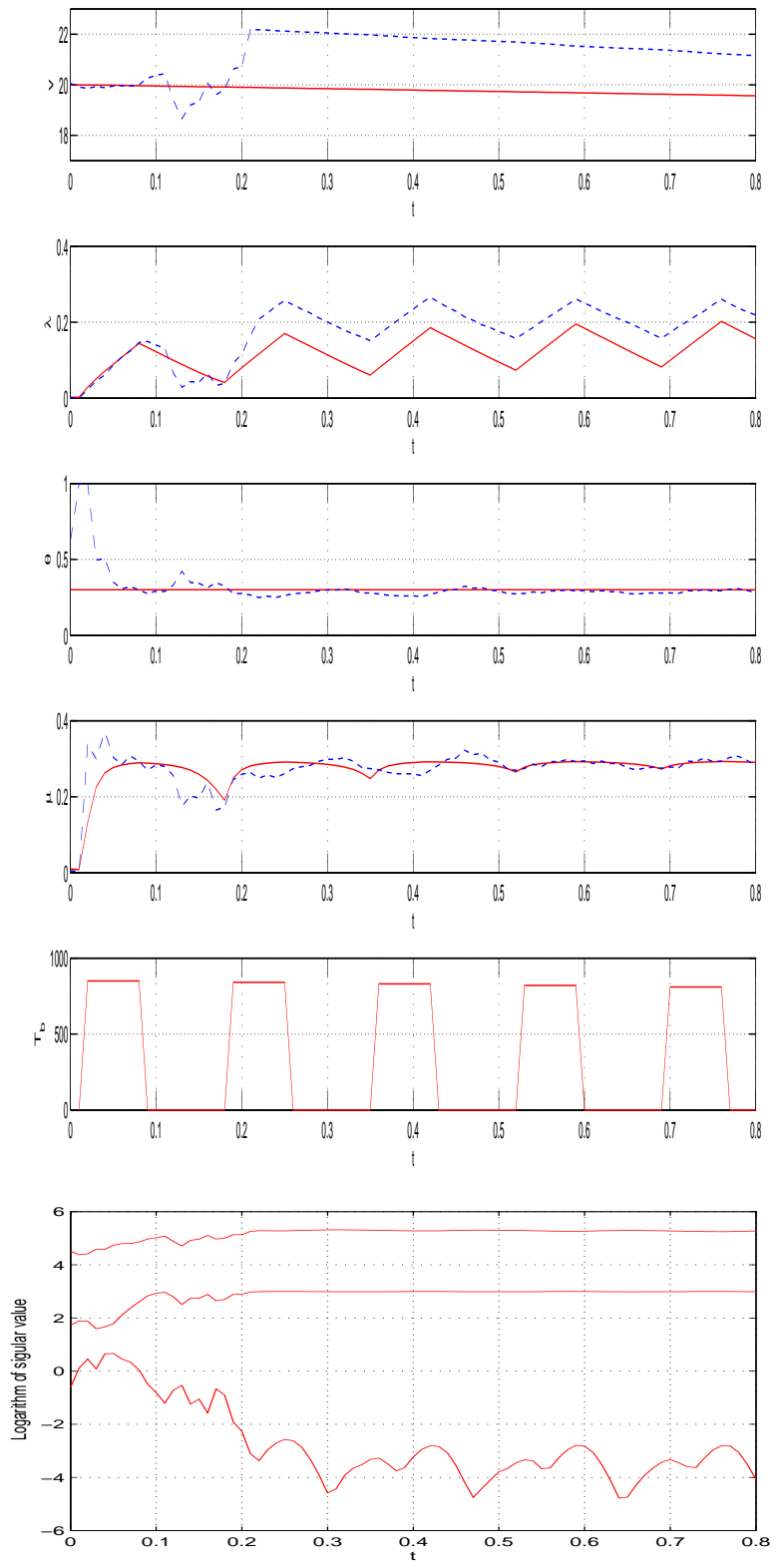

Fig. 4. Simulation results of case 2 .

- Case 3, for dry asphalt, the simulation result is shown in Figure 5.

- Case 4, for snow, the simulation result is shown in Figure 6.

From the figures, the example illustrates there are some potential benefits of estimating more parameters $B, C, E$ than $\theta$. With reference to Figure 2 it is clear that in the low friction case (snow) the friction curve has no distinct peak such that the parameters $E$ and $B$ are poorly observable independently of persistence of excitation, unlike the high friction case (dry asphalt) where all parameters of the Magic formula have some influence on the curve shape and will be observable with persistence of excitation. It is interesting
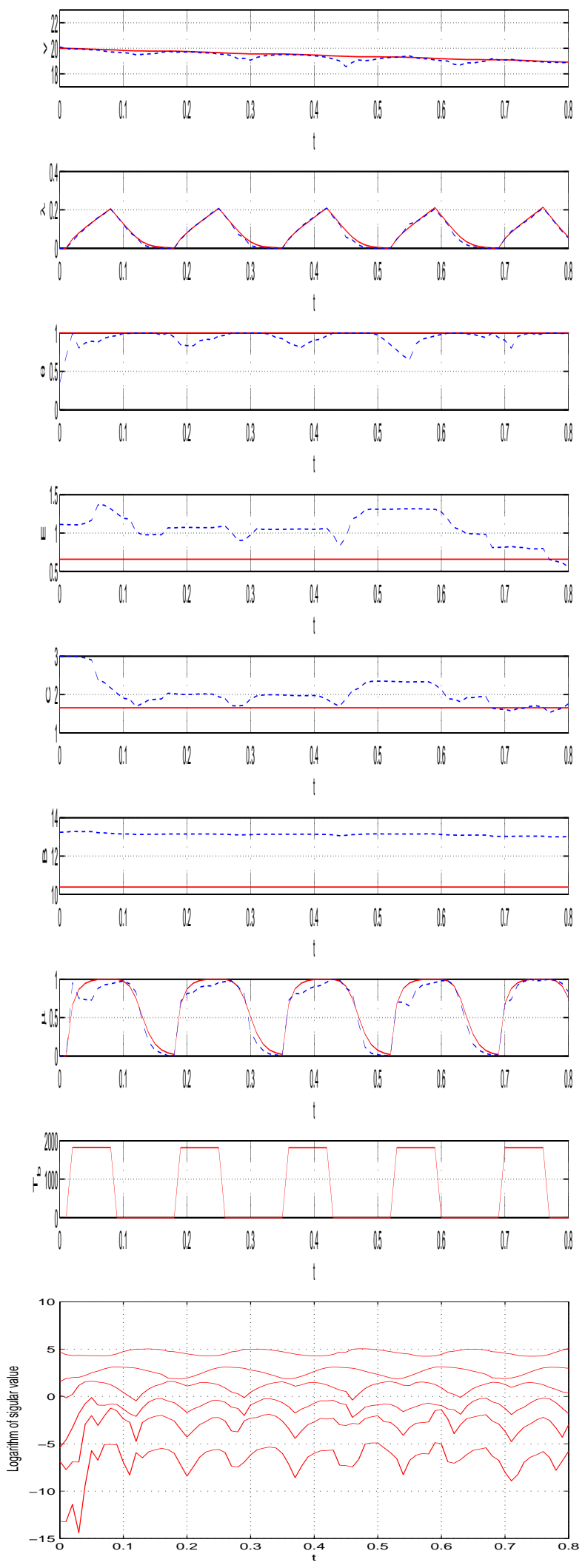

Fig. 5. Simulation results of case 3. 

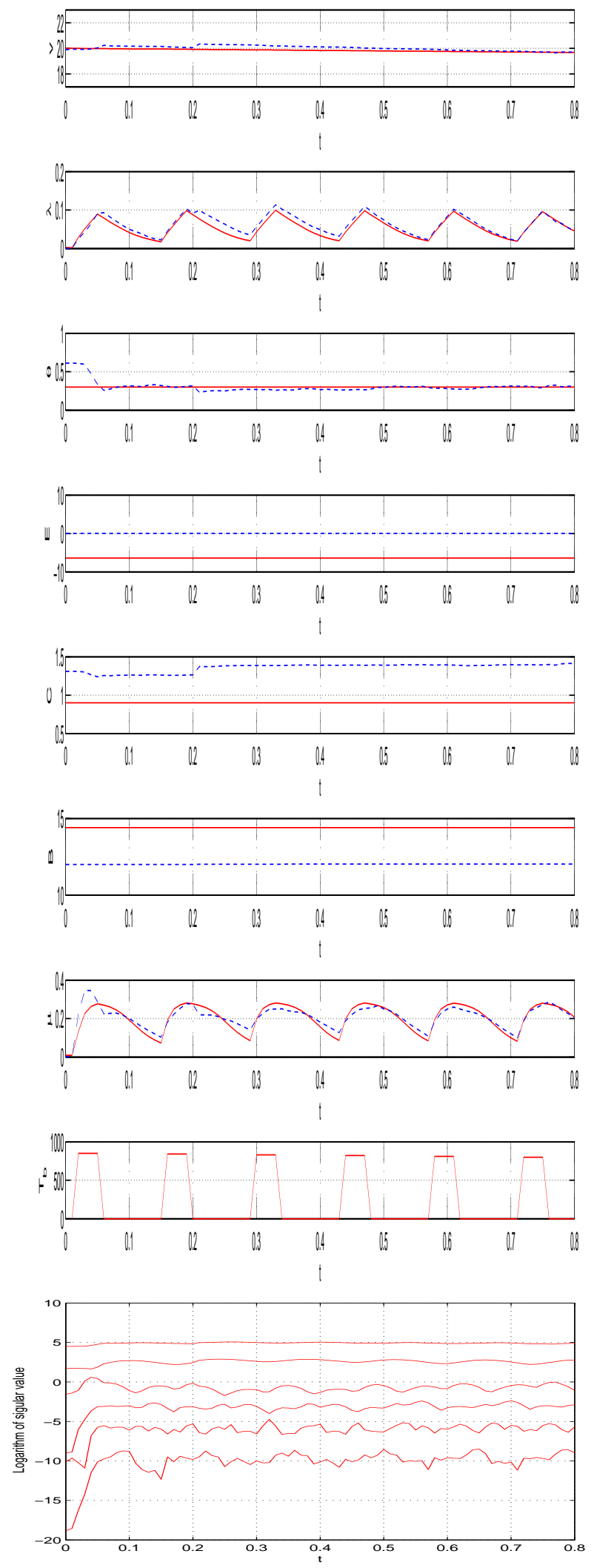

Fig. 6. Simulation results of case 4 . to observe that the estimation is robust and that the SVD thresholding effectively prevents the estimates of $(B, C, E)$ from drifting and becoming highly incorrect when there is not much excitation or they are poorly observable.

\section{CONCLUSION}

In this paper MHE for ABS has been proposed for combined state and parameter estimation problem. The main feature of the proposed MHE algorithm is handling of nonlinear systems that are neither uniformly observable, nor persistently excited. The mechanisms to handle unobservable states, parameters and non-informative data are classical tools of regularization such as penalty terms with a priori information, and the use of the thresholded singular value decomposition. In the example it is illustrated that the regularized MHE method can deal with situations when the friction model is over-parameterized (in the sense that some parameters are poorly observable) or there is lack of excitations.

\section{REFERENCES}

[1] M. Burckhardt. Fahrwerktechnik: Radschlupf-regelsysteme. Vogel Verlag, 1993.

[2] SAE. Antilock brake review. Tech. Rep. J2246, Society of Automative Engineerings, Warrendale PA, 1992.

[3] T. A. Johansen, I. Petersen, J. Kalkkuhl, and J. Ludemann. Gainscheduled wheel slip control in automotive brake systems. IEEE Trans. Control Systems Technology, 11:799-811, 2003.

[4] L. Alvarez, J. Yi, R. Horowitz, and L. Olmos. Dynamic friction modelbased tire-road friction estimation and emergency braking control Transactions of the ASME, 127:22-32, 2005.

[5] C. Canudas de Wit, R. Horowitz, and P. Tsiotras. Model-based observers for tire/road contact friction prediction. In New Directions in nonlinear observer design, volume 244. Lecture Notes in Control and Information Sciences, Springer Berlin, 1999.

[6] L. Alvarez and R. Horowitz. Adaptive emergency braking control with understanding of friction coffcient. IEEE Trans. Control Systems Technology, 10:381-392, 2002.

[7] N. Patel, C. Edwards, and S. K. Spurgeon. Tyre-road friction estimation - a comparative study. IMechE, Part D: Automotive Engineering, 222:2337-2351, 2008.

[8] U. Kiencke. Real-time estimation of adhesion characteristic between tyre and road. In Proceedings of the IFAC World Congress, Sydney, 1993.

[9] K. Li, J. A. Misener, and K. Hedrick. On-board road condition monitoring system using slip-based tyre-road friction estimation and wheel speed signal analysis. IMechE, Part K: Multi-body dynamics, 221:129-146, 2007.

[10] J. Matuško, I. Petrović, and N. Perić. Application of extended kalman filter for road condition estimation. Automatica, 44:59-65, 2003.

[11] E. Bakker, L. Nyborg, and H. Pacejka. Tyre modelling for use in vehicle dynamics studies. page SAE paper 870321. Society of Automotive Engineering, 1987.

[12] P. E. Moraal and J. W. Grizzle. Observer design for nonlinear systems with discrete-time measurement. IEEE Transactions Automatic Control, 40:395-404, 1995.

[13] D. Sui and T. A. Johansen. Regularized moving horizon observer for detectable nonlinear systems. In IFAC Symposium on Nonlinear Control, Bologna, Italy, 2010.

[14] A. Alessandri, M. Baglietto, and G. Battistelli. Moving-horizon state estimation for nonlinear discrete-time systems: New stability results and approximation schemes. Automatica, 44:1753-1765, 2008.

[15] Z. Wang, Z. Liu, and R. Pei. Estimation of vehicle speed and friction force using moving horizon strategy. In Proc. 5th IEEE World Congress on Intelligent Control and Automation, Hangzhou, China, pages $1547-1550,2004$.

[16] G. H. Golub and C. F. van Loan. Matrix computations. Oxford University Press, 1983. 